ORIGINAL ARTICLE

\title{
Cytomegalovirus infection of the cervix: morphological observations in five cases of a possibly under-recognised condition
}

\author{
C E McGalie, H A McBride, W G McCluggage
}

J Clin Pathol 2004;57:691-694. doi: 10.1136/icp.2004.016162

See end of article for authors' affiliations

.....................

Correspondence to: Dr W G McCluggage, Department of Pathology, Royal Group of Hospitals Trust, Grosvenor Road Belfast BT1 2 6BL, Northern Ireland; glenn. mccluggage@bill.n-i.nhs.uk

Accepted for publication 6 February 2004
Aims: Histologically diagnosed cytomegalovirus (CMV) infection of the cervix is rare and the associated morphological features are not well described. This study describes histopathological findings in five biopsies from four patients with CMV cervicitis.

Methods: CMV inclusions were identified in five cervical biopsies from four patients in a single institution over eight months. The clinical notes were reviewed, the morphological features documented, and immunohistochemical staining for CMV performed. CMV immunohistochemical staining was also performed on 30 consecutive cervical biopsies in which inclusions were not seen histologically.

Results: None of the patients was immunocompromised but one was postnatal. Numbers of CMV inclusions ranged from occasional to abundant and they were located mainly in endocervical glandular epithelial cells but also in endothelial and mesenchymal cells. Inclusions were not seen in squamous cells. Inclusions were eosinophilic and were intracytoplasmic rather than intranuclear. They were positive immunohistochemically for CMV. Associated morphological features included fibrin thrombi within small blood vessels (three cases), dense active inflammatory infiltrates (five cases), lymphoid follicles (two cases), vacuolation of glandular epithelial cells (two cases), and reactive changes in glandular epithelial cells (two cases). CMV inclusions were not identified in the 30 additional cases that underwent immunohistochemical staining.

Conclusions: CMV infection of the cervix may be more common than is thought. Patients are usually immunocompetent and require no treatment. Morphological features such as a dense inflammatory cell infiltrate with lymphoid follicles, and especially fibrin thrombi within small vessels, should alert the pathologist to look closely for the pathognomonic CMV inclusion bodies.
$\mathrm{H}$ istologically diagnosed cytomegalovirus (CMV) infection of the cervix is rare and the published literature is limited to a few reports, mainly of individual cases or small numbers of cases. ${ }^{1-5}$ In this report, we describe the histological features in five biopsies from four patients with CMV infection of the cervix diagnosed in a single institution over an eight month period. By documenting the associated histological appearances we hope to identify features that should alert the pathologist to search for CMV inclusion bodies. We also reviewed the clinical notes to ascertain whether factors predisposing to CMV infection were present and performed CMV immunohistochemical staining on 30 consecutive cervical biopsies where inclusions were not seen to determine whether this might identify additional cases that were not diagnosed on morphological examination.

\section{MATERIALS AND METHODS}

Cases were reported by a gynaecological pathologist (WGM) in a single institution over an eight month period. The five cases of CMV cervicitis were from a total of 957 cervical biopsies reported during that period. The associated morphological features were documented.

\section{Immunohistochemistry}

The five cases with morphologically identified CMV inclusions underwent immunohistochemical staining with anti-CMV antibody (Dako, Ely, Cambridgeshire, UK). Immunolocalisation was performed using an automated staining machine (Ventana Nexes, Strasbourg, France). In addition, 30 consecutive cervical biopsies, where there was no morphological evidence of CMV infection, were stained with anti-CMV antibody.

\section{RESULTS}

The age of the women ranged from 19 to 30 years. All patients had been referred for colposcopy following cervical smears showing borderline nuclear changes or dyskaryosis. CMV inclusions were not identified in the cervical smears. All patients were asymptomatic. Two had a past history of a sexually transmitted disease and one was six months postnatal. None of the patients had a known history of CMV infection and none was immunocompromised or on immunosuppressant treatment. None of the patients had received a recent blood transfusion.

The specimens comprised three punch biopsies and two large loop excisions of the transformation zone (LLETZ). One patient had both a punch and a LLETZ biopsy.

CMV inclusions were sparse in two of the five biopsies and abundant and easily identified in the other three. In all cases, inclusions were eosinophilic and intracytoplasmic, and were mainly located within the glandular epithelial cells (fig 1). In four cases, inclusions were also present within endothelial cells (fig 2) and in three cases in mesenchymal stromal cells. Inclusions were not identified in squamous epithelial cells.

All five biopsies contained a dense active inflammatory cell infiltrate largely composed of neutrophils, lymphocytes, and plasma cells (fig 3 ). Well formed lymphoid follicles were seen in two cases (fig 4) and intracytoplasmic vacuoles containing

Abbreviations: $\mathrm{CIN}$, cervical intraepithelial neoplasia; CMV, cytomegalovirus; LLETZ, large loop excision of the transformation zone 


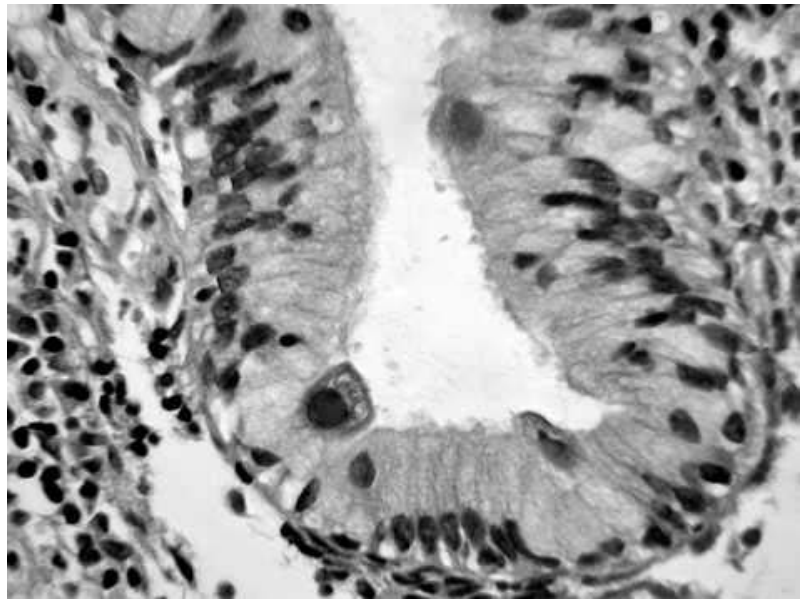

Figure 1 Intracytoplasmic cytomegalovirus inclusions within endocervical glandular epithelial cells.

neutrophils were present within endocervical cells in two cases (fig 5). Reactive glandular epithelial change with mild nuclear atypia was identified in two cases. An additional prominent feature noted in three cases was the presence of fibrin thrombi within many small capillary sized stromal blood vessels (fig 6) without evidence of vasculitis. In all cases, the inclusions stained positively with anti-CMV antibody (fig 7).

Other findings in the biopsies included koilocytosis in all cases, cervical intraepithelial neoplasia 1 (CIN 1) in two cases, and CIN 3 in two cases. CMV inclusions were not identified in the 30 additional cases that underwent immunohistochemical staining.

\section{DISCUSSION}

Using sensitive methods, such as in situ hybridisation and polymerase chain reaction, CMV can be identified in the cervix in a considerable proportion of women. ${ }^{6-10}$ Rates of CMV infection of up to $29 \%$ have been found in women with normal cervical smears. ${ }^{8}$ Rates of detection are higher in younger women, ${ }^{6}$ in those with human papillomavirus infection, ${ }^{9}$ and in prostitutes and women attending sexually transmitted disease clinics. ${ }^{7}$ However, histologically diagnosed CMV cervicitis is rare, with reports limited to individual cases or small numbers of cases..$^{1-5}$ In addition, CMV

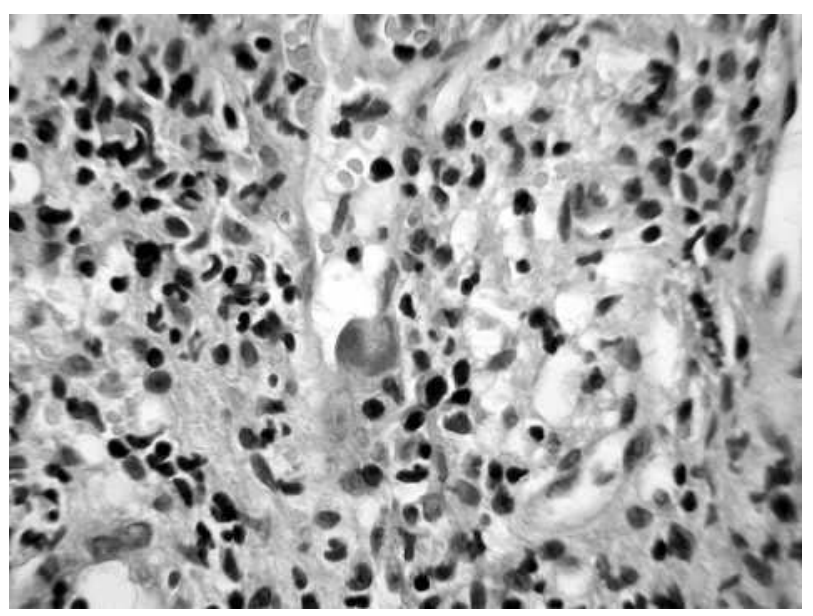

Figure 2 Intracytoplasmic cytomegalovirus inclusions within endothelial cells.

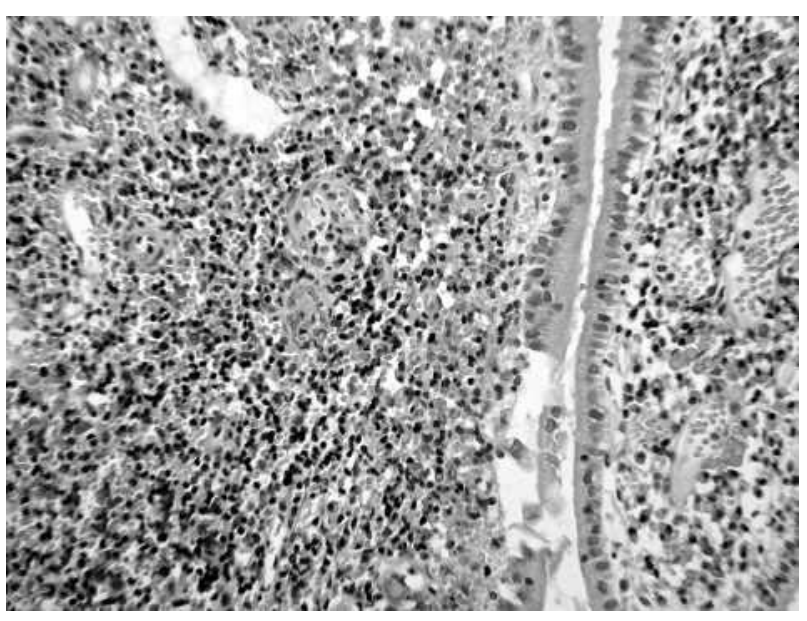

Figure 3 Case showing a pronounced active inflammatory cell infiltrate.

inclusions have been detected rarely in cervical smears, ${ }^{1}{ }^{11}$ although in our cases of histologically confirmed CMV infection, inclusion bodies were not seen in the corresponding cervical smears. The finding of CMV inclusion bodies in five cervical biopsies over a short time period suggests that CMV infection of the cervix may be more common than is generally appreciated, and that a high index of suspicion is required to diagnose this. However, the occurrence of five cases in a short time period may be coincidental because no cases of CMV cervicitis had been diagnosed in our institution over the preceding 18 years.

We reviewed the associated histological features with the aim of documenting the morphological appearances that should alert the pathologist to the possibility of CMV infection. Inclusions were seen mainly in the endocervical glandular epithelial cells but also in endothelial and mesenchymal cells. Inclusions were not identified in squamous cells. This predominant location of inclusion bodies in glandular epithelial cells is in keeping with the findings in previously reported cases of CMV cervicitis. ${ }^{1245}$ When CMV involves other organs, inclusions are usually intranuclear, but in our cases of CMV cervicitis inclusions were intracytoplasmic. This was most easily appreciated in endocervical cells where the intracytoplasmic location was readily apparent. A common feature in all five biopsies was the presence of a dense active inflammatory cell infiltrate, largely composed of

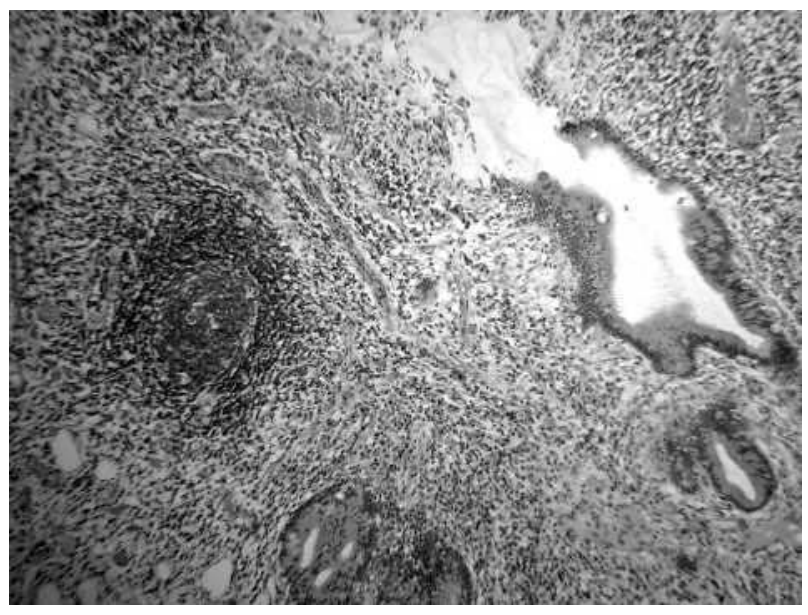

Figure 4 Case containing well formed lymphoid follicles. 


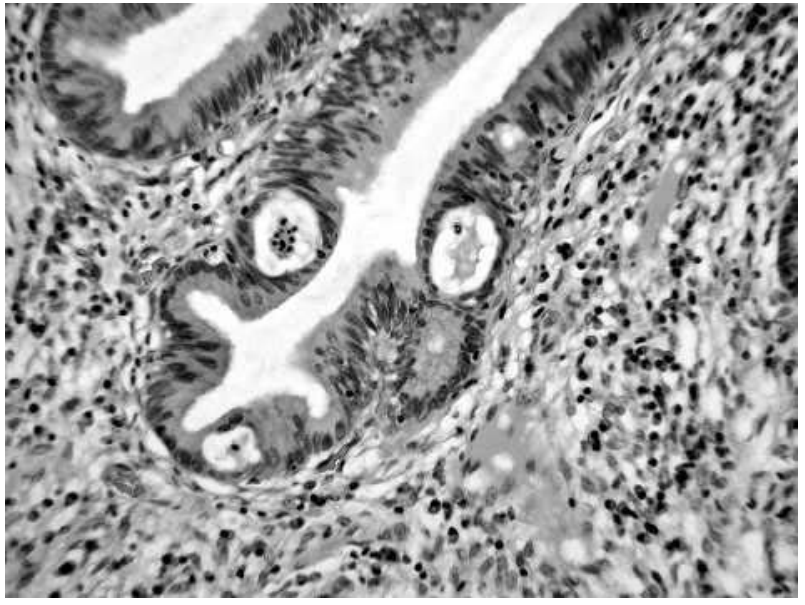

Figure 5 Intracytoplasmic vacuoles containing neutrophils within endocervical glandular epithelial cells.

neutrophils, lymphocytes, and plasma cells. Lymphoid follicles were present in two cases. Although a non-specific finding, the presence of lymphoid follicles has been described previously in CMV cervicitis. ${ }^{4}$ In two cases, there was reactive nuclear atypia of glandular epithelial cells, presumably secondary to the associated inflammation, and in two cases the glandular epithelial cells contained intracytoplasmic vacuoles with neutrophils. An additional unusual feature that has not, to the best of our knowledge, been described previously was the presence of fibrin thrombi within small capillary sized blood vessels in three cases. In our experience, this is unusual in the cervix and we feel that this feature should alert the pathologist to look closely for the pathognomonic CMV inclusion bodies, and further levels should be cut when inclusions are not seen initially. It is probable that the fibrin thrombi are secondary to CMV infection of endothelial cells. Vascular thrombosis has rarely been described in immunocompetent patients with acute CMV infection, and in those who are immunosuppressed..$^{12}$ The absence of immunohistochemical staining in the 30 additional cases suggests that active CMV infection (either primary infection or reactivation) of the cervix does not occur in the absence of histologically demonstrable inclusion bodies although, as exemplified by some of our cases, these may be sparse.

CMV infection of sites in the female genital tract other than the cervix has also been reported rarely. Abulafia et al described CMV inclusion bodies in a recurrent ulcerative

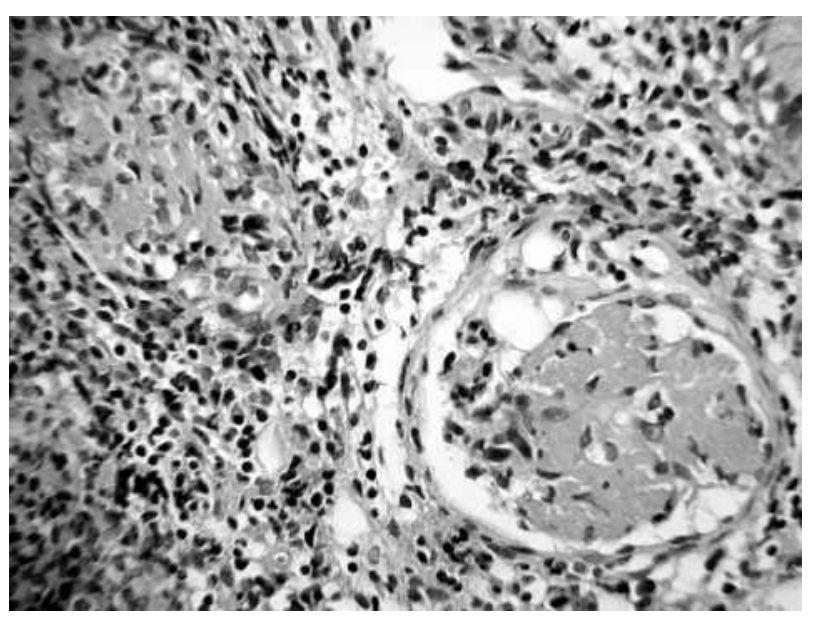

Figure 6 Fibrin thrombi within small stromal blood vessels.

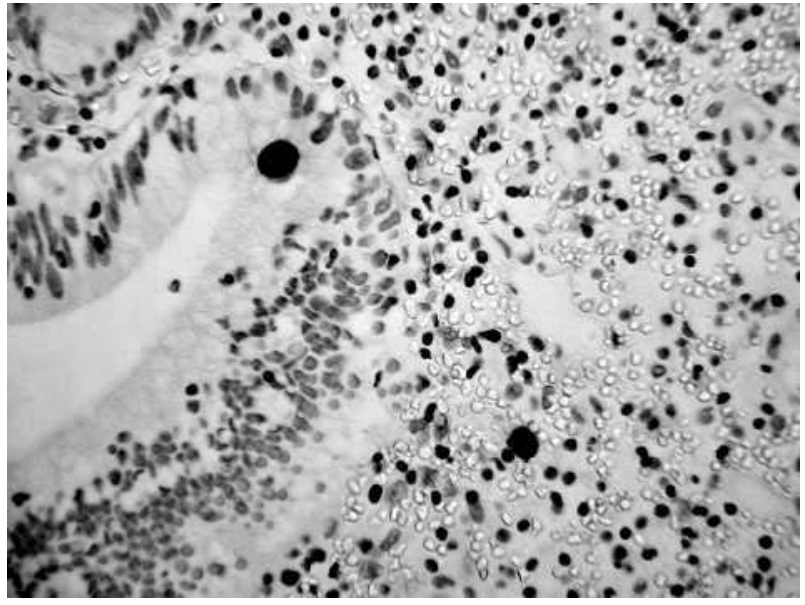

Figure 7 Positive immunohistochemical staining involving glands and stroma with cytomegalovirus.

vaginal lesion, ${ }^{13}$ and cases of CMV endometritis have also been reported..$^{4} 14{ }^{15}$ Some of these cases of CMV endometritis were associated with a lymphoplasmacytic infiltrate with germinal centre formation within the endometrium, ${ }^{4}$ and granulomatous inflammation has also been described. ${ }^{14}$ In addition, a case of disseminated CMV infection of the female genital tract with involvement of the vulva, vagina, and cervix has been described in a patient with AIDS, ${ }^{3}$ in addition to occasional cases of CMV oophoritis ${ }^{16}$ and of vulval involvement. ${ }^{18}$

"Absence of immunohistochemical staining in the 30 additional cases suggests that active cytomegalovirus infection of the cervix does not occur in the absence of histologically demonstrable inclusion bodies although these may be sparse"

Most of the previous reports of CMV cervicitis have been in asymptomatic, immunocompetent patients. However, CMV cervicitis has also been described in immunosuppressed patients, including women with AIDS, ${ }^{3}{ }^{19}$ and those who are immunocompromised for other reasons. ${ }^{1}$ The four patients in our series were asymptomatic, having been referred for colposcopy because of abnormal cervical smears, and were not immunocompromised. However, two had a history of sexually transmitted diseases and one was postnatal. It has been suggested that pregnancy can result in reactivation of CMV as a result of hormonal stimuli. ${ }^{20}{ }^{21}$ Whether infection in our cases represents primary involvement of the cervix by CMV or reactivation of latent virus is impossible to ascertain. When the patient is asymptomatic and immunocompetent, no treatment of CMV cervicitis is required and the infection is unlikely to be clinically relevant. However, infection may be important in immunocompromised women with gynaecological symptoms. In addition, in pregnant women CMV infection may result in early spontaneous abortion and there is a risk of fetal infection, which may result in congenital abnormalities. The classic triad of congenital CMV infection consists of chorioretinitis, calcification of the cerebrum, and microcephaly. ${ }^{22}$ Other common features in newborn babies include jaundice, haemolytic anaemia, pneumonitis, thrombocytopenic purpura, and hydrocephaly.

In summary, we describe the histological features in five biopsies from four patients with CMV cervicitis. We suggest that CMV infection of the cervix may be more common than is generally appreciated. Histological features such as a dense 


\section{Take home messages}

- Cytomegalovirus (CMV) infection of the cervix may be more common than is generally thought

- Histological features such as a dense inflammatory infiltrate with lymphoid follicles, and especially fibrin thrombi within small blood vessels, should alert the pathologist to look closely for the pathognomonic CMV inclusion bodies

inflammatory infiltrate with lymphoid follicles, and especially fibrin thrombi within small blood vessels, should alert the pathologist to look closely for the pathognomonic CMV inclusion bodies.

\section{ACKNOWLEDGEMENTS}

We would like to thank Dr S Dobbs (gynaecologist) and Dr M Mulholland (gynaecologist) for providing the clinical details of these patients.

\section{Authors' affiliation}

C E McGalie, H A McBride, W G McCluggage, Department of Pathology, Royal Group of Hospitals Trust, Belfast BT12 6BL, Northern Ireland, UK

\section{REFERENCES}

1 Huang JC, Naylor B. Cytomegalovirus infection of the cervix detected by cytology and histology: a report of five cases. Cytopathology 1993;4:237-41.

2 Byard RW, Mikhael NZ, Orlando G, et al. The clinicopathological significance of cytomegalovirus inclusions demonstrated by endocervical biopsy. Pathology $1991 ; 23: 318-21$

3 Friedmann W, Schafer A, Kretschmer R, et al. Disseminated cytomegalovirus infection of the female genital tract. Gynecol Obstet Invest 1991;31:56-7.

4 Wenckebach GFC, Curry B. Cytomegalovirus infection of the female genital tract. Histologic findings in three cases and review of the literature. Arch Pathol Lab Med 1976;100:609-12.
5 Ross L. Incidental finding of cytomegalovirus inclusions in cervical glands. Am J Obstet Gynecol 1966;95:956-8.

6 Gradilone A, Vercillo R, Napolitano M, et al. Prevalence of human papillomavirus, cytomegalovirus and Epstein-Barr virus in the cervix of healthy women. J Med Virol 1996;50:1-4.

7 Shen CY, Chang SF, Lin HJ, et al. Cervical cytomegalovirus infection in prostitutes and in women attending a sexually transmitted disease clinic. J Med Virol 1994:43:362-6.

8 Schon HJ, Schurz B, Marz R, et al. Screening for Epstein-Barr and human cytomegalovirus in normal and abnormal cervical smears by fluorescent in situ cytohybridization. Arch Virol 1992;125:205-14.

9 Mougin C, Schaal JP, Bassignot A, et al. Detection of human papillomavirus and human cytomegalovirus in cervical lesions by in situ hybridization using biotinylated probes. Biomed Pharmacother 1991;45:353-7.

10 Yuan CF, Kao SM, Wang DC, et al. Detection of human cytomegalovirus in cervicovaginal cells by culture, in situ DNA hybridization and DNA amplification methods. Mol Cell Probes 1990;4:475-83.

11 Gideon K, Zaharopoulos P. Cytomegalovirus endocervicitis diagnosed by cervical smear. Diagn Cytopathol 1991;7:625-7.

12 Abgueguen $\mathbf{P}$, Delbos V, Chennebault JM, et al. Vascular thrombosis and acute cytomegalovirus infection in immunocompetent patients: report of 2 cases and literature review. Clin Infect Dis 2003;36:E134-9.

13 Abulafia O, Du Beshter B, Dawson AE, et al. Presence of cytomegalovirus inclusion bodies in a recurrent ulcerative vaginal lesion. Am J Obstet Gynecol 1993;169:1179-80.

14 McCracken AW, D'Agnostino AW, Brucks AB, et al. Acquired cytomegalovirus infection presenting as viral endometritis. Am J Clin Pathol 1974:61:556-60.

15 Frank TS, Himebaugh KS, Wilson MD. Granulomatous endometritis associated with histologically occult cytomegalovirus in a healthy patient. Am J Surg Pathol 1992;16:716-20.

16 Iwasaki T, Sakuma T, Satodate R, et al. Cytomegalovirus oophoritis with cortical necrosis during remission of acute lymphocytic leukaemia. Acta Pathol Jpn 1988;38:1069-76.

17 LiVolsi SA, Merino MJ. Cytomegalovirus infection of ovarian thecoma. Arch Pathol Lab Med 1979;103:653-4.

18 Chetty R, Bramdev A, Govender D. Cytomegalovirus-induced syringosquamous metaplasia. Am J Dermatopathol 2000;22:559-61

19 Brown S, Senekjian EK, Montag AG. Cytomegalovirus inclusions of the uterine cervix in a patient with acquired immunodeficiency syndrome. Obstet Gynecol 1988;71:489-91.

20 Montgomery R, Youngblood L, Medearis DN. Recovery of cytomegalovirus from the cervix in pregnancy. Pediatrics 1972;49:524-31.

21 Reynolds DW, Stagno S, Hosty TS, et al. Maternal cytomegalovirus excretion and perinatal infection. N Engl J Med 1973;289:1-5.

22 Nankervis GA, Bhumbra NA. Cytomegalovirus infection of neonate and infant. Adv Pediatr Infect Dis 1986;1:61-74. 